\title{
Probing the unitarity of the renormalizable theory of massive conformal gravity
}

\author{
F. F. Faria ${ }^{1, \mathrm{a}}$ \\ ${ }^{1}$ Centro de Ciências da Natureza, Universidade Estadual do Piauí, 64002-150 Teresina, PI, Brazil
}

Received: 15 July 2017 / Accepted: 26 March 2018 / Published online: 31 March 2018

(C) The Author(s) 2018

\begin{abstract}
The presence of an unstable massive spin-2 ghost state in the renormalizable theory of massive conformal gravity leads to a pair of complex poles appearing in the first sheet of the energy plane. Here we show that the positions of these poles are gauge dependent, which makes the theory unitary.
\end{abstract}

Keywords Gauge symmetry · Unitarity · Quantum Gravity

\section{Introduction}

The massive conformal gravity (MCG) is a renormalizable theory of gravity $[1,2]$ that has, in addition to the usual positive energy massless spin-2 field, a negative energy massive spin-2 field. At the quantum level, the negative energy field translates into an unstable negative norm ghost state. The instability of the ghost state makes it necessary to use a modified perturbation expansion in terms of dressed propagators. Since the bare propagator of the ghost state has a negative residue, the original real pole split into a pair of complexconjugate poles in the first Riemannian energy sheet of the dressed propagator. If the positions of the complex poles are gauge dependent, we can move them around by varying the corresponding gauge-fixing parameter. In this case, the $S$ matrix connects only asymptotic states with positive norm and thus it is a unitary matrix.

It is well known that fourth order derivative theories of gravity have a massive ghost pole (or rather complex poles in the dressed propagator) whose position is gauge independent [3]. The advantage of MCG over these theories is that its linearized action is invariant, independently, under coordinate and conformal gauge transformations. Thus, even if the position of the MCG massive ghost pole is independent of the coordinate gauge-fixing parameter, as happens in the fourth order derivative theories of gravity, its dependence on

a e-mail: felfrafar@hotmail.com the conformal gauge-fixing parameter alone is sufficient to ensure the unitarity of the theory.

This paper is organized as follows. In Sect. 2, we describe the nature of the MCG massive ghost pole. In Sect. 3, we probe the gauge dependence of the positions of the MCG complex poles by using the Nielsen identities. In Sect. 4, we present our conclusions.

\section{Massive ghost pole}

We start by considering the MCG action ${ }^{1}$ [4]

$$
\begin{aligned}
S_{\mathrm{MCG}}= & \int d^{4} x \mathcal{L}_{\mathrm{MCG}} \\
= & \frac{1}{k^{2}} \int d^{4} x \sqrt{-g}\left[\varphi^{2} R+6 \partial_{\mu} \varphi \partial^{\mu} \varphi\right. \\
& \left.-\frac{1}{2 m^{2}} C^{\alpha \beta \mu \nu} C_{\alpha \beta \mu \nu}\right],
\end{aligned}
$$

where $k^{2}=16 \pi G, m$ is a constant with dimension of mass, $\varphi$ is a scalar field called dilaton,

$$
\begin{aligned}
C^{\alpha \beta \mu \nu} C_{\alpha \beta \mu \nu}= & R^{\alpha \beta \mu \nu} R_{\alpha \beta \mu \nu}-4 R^{\mu v} R_{\mu \nu}+R^{2} \\
& +2\left(R^{\mu v} R_{\mu \nu}-\frac{1}{3} R^{2}\right)
\end{aligned}
$$

is the Weyl tensor squared, $R_{\mu \beta \nu}^{\alpha}$ is the Riemann tensor, $R_{\mu \nu}=R_{\mu \alpha \nu}^{\alpha}$ is the Ricci tensor and $R=g^{\mu \nu} R_{\mu \nu}$ is the scalar curvature.

Using the Lanczos identity, performing the background field expansions

$$
\begin{aligned}
g_{\mu \nu} & =\eta_{\mu \nu}+k h_{\mu \nu}, \\
\varphi & =1+k \sigma,
\end{aligned}
$$

and keeping only the terms of second order in the fields $h_{\mu \nu}$ and $\sigma$, it can be shown that the linear approximation of the MCG Lagrangian density (1) is given by [1]

\footnotetext{
$\overline{1}$ Here we use units in which $c=\hbar=1$.
} 


$$
\begin{aligned}
\overline{\mathcal{L}}_{\mathrm{MCG}}= & \overline{\mathcal{L}}_{E H}+2 \sigma \bar{R}+6 \partial^{\mu} \sigma \partial_{\mu} \sigma \\
& -\frac{1}{m^{2}}\left(\bar{R}^{\mu \nu} \bar{R}_{\mu \nu}-\frac{1}{3} \bar{R}^{2}\right),
\end{aligned}
$$

where

$\bar{R}_{\mu \nu}=\frac{1}{2}\left(\partial_{\mu} \partial^{\rho} h_{\rho \nu}+\partial_{\nu} \partial^{\rho} h_{\rho \mu}-\square h_{\mu \nu}-\partial_{\mu} \partial_{\nu} h\right)$

is the linearized Ricci tensor,

$\bar{R}=\partial^{\mu} \partial^{v} h_{\mu \nu}-\square h$

is the linearized scalar curvature, and

$$
\begin{aligned}
\overline{\mathcal{L}}_{E H}= & -\frac{1}{4}\left(\partial^{\rho} h^{\mu \nu} \partial_{\rho} h_{\mu \nu}-2 \partial^{\mu} h^{\nu \rho} \partial_{\rho} h_{\mu \nu}\right. \\
& \left.+2 \partial^{\mu} h_{\mu \nu} \partial^{\nu} h-\partial^{\mu} h \partial_{\mu} h\right)
\end{aligned}
$$

is the linearized Einstein-Hilbert Lagrangian density, with $\square=\eta^{\mu \nu} \partial_{\mu} \partial_{\nu}$ and $h=\eta^{\mu \nu} h_{\mu \nu}$.

The linearized Lagrangian density (5) is invariant under the coordinate gauge transformation

$h_{\mu \nu} \rightarrow h_{\mu \nu}+\partial_{\mu} \chi_{\nu}+\partial_{\nu} \chi_{\mu}$,

where $\chi^{\mu}$ is an arbitrary spacetime dependent vector field, and under the conformal gauge transformations

$h_{\mu \nu} \rightarrow h_{\mu \nu}+2 \eta_{\mu \nu} \Lambda, \quad \sigma \rightarrow \sigma-\Lambda$,

where $\Lambda$ is an arbitrary spacetime dependent scalar field. In order to fix these gauge freedoms, we must add the gaugefixing terms

$\mathcal{L}_{G F 1}=-\frac{1}{2 \xi_{1}}\left(\partial^{\mu} h_{\mu \nu}-\frac{1}{2} \partial_{\nu} h\right)^{2}$,

$\mathcal{L}_{G F 2}=\frac{1}{6 \xi_{2}}\left(\frac{1}{m} \bar{R}-6 m \xi_{2} \sigma\right)^{2}$,

to (5), where $\xi_{1}$ and $\xi_{2}$ are gauge-fixing parameters.

Using the Barnes-Rivers projectors [5,6], and performing a long but straightforward calculation, we can show that the gauge-fixed Lagrangian density $\overline{\mathcal{L}}_{\mathrm{MCG}}+\mathcal{L}_{G F 1}+\mathcal{L}_{G F 2}$ leads to the spin-2 part of the graviton bare propagator

$D_{\mu \nu, \alpha \beta}^{(2)}=-i\left[\frac{1}{p^{2}}-\frac{1}{p^{2}+m^{2}}\right] P_{\mu \nu, \alpha \beta}^{(2)}$

where

$P_{\mu \nu, \alpha \beta}^{(2)}=\frac{1}{2}\left(\theta_{\mu \alpha} \theta_{\nu \beta}+\theta_{\mu \beta} \theta_{\nu \alpha}\right)-\frac{1}{3} \theta_{\mu \nu} \theta_{\alpha \beta}$

is the spin-2 projector, with $\theta_{\mu \nu}=\eta_{\mu \nu}-p_{\mu} p_{\nu} / p^{2}$. The first term in the brackets in (13) is the usual massless graviton pole at $p^{2}=0$ with positive residue, and the second is a massive ghost pole at $p^{2}=-m^{2}$ with negative residue. The massive ghost is, however, unstable because its mass is above the normal threshold of the massless graviton production. Thus, since the ordinary perturbation theory breaks down near the mass of an unstable particle [7], we must use a modified perturbation series in which the bare propagator $D\left(p^{2}\right)$ is replaced by the dressed propagator [8]

$\bar{D}\left(p^{2}\right)=\left[D^{-1}\left(p^{2}\right)-\Pi\left(p^{2}\right)\right]^{-1}$,

where $\Pi\left(p^{2}\right)$ is the sum of all one-particle irreducible (1PI) self-energy parts.

By coupling $N$ fermion fields to the MCG action (1), expanding in powers of $1 / N$, and using the Cauchy integral theorem, we can write the spin-2 part of the graviton dressed propagator in the spectral form [9]

$$
\begin{aligned}
\bar{D}_{\mu \nu, \alpha \beta}^{(2)}= & -i\left[\frac{1}{p^{2}}+\frac{\mathscr{R}}{p^{2}-M^{2}}+\frac{\mathscr{R}^{*}}{p^{2}-M^{* 2}}\right. \\
& \left.+\frac{1}{2 \pi} \int_{C} \frac{\rho(a)}{p^{2}-a} d a\right] P_{\mu \nu, \alpha \beta}^{(2)}+\text { gauge terms, }
\end{aligned}
$$

where $M, M^{*}, \mathscr{R}$, and $\mathscr{R}^{*}$ are the positions and residues of a complex-conjugate pole pair, respectively, $\rho(a)$ is a spectral function obtained by cutting all the self-energy graphs of the continuum states, and $C$ is an appropriate path in the complex plane. We can see from (16) that the pole for the unstable massive ghost has split into a pair of complex-conjugate poles in the physical Riemannian energy sheet, which supposedly breaks the unitarity of the $S$-matrix. However, if the positions of the complex poles are gauge dependent, the unitarity of the gauge-invariant $S$-matrix is satisfied. We will address this issue in the next section.

\section{Gauge dependence}

It is well known that, for the position of a massive pole $m_{\text {pole }}^{2}$ to be gauge independent, it must satisfy the Nielsen identity [10]

$\xi \frac{\partial m_{\text {pole }}^{2}}{\partial \xi}+C(\widehat{\phi}, \xi) \frac{\partial m_{\text {pole }}^{2}}{\partial \widehat{\phi}}=0$,

where $\xi$ is any gauge-fixing parameter, the hat represents setting all the fields of the theory, denoted generically by $\phi$, to their vacuum expectation values, and $C(\widehat{\phi}, \xi)$ can be determined order by order in the loop expansion of the theory.

In order to derive the Nielsen identities for the MCG complex poles, we choose the Becchi-Rouet-Stora-Tyutin (BRST) method [11-14], which consists in the inclusion of ghost and anti-ghost fields arising from the gauge redundancies of the theory [15]. The addition of the compensating Faddeev-Popov ghost terms

$$
\begin{aligned}
\mathcal{L}_{F P 1} & =\tilde{c}^{\mu} \square c_{\mu}, \\
\mathcal{L}_{F P 2} & =2 \tilde{c}\left(\square-\xi_{2} m^{2}\right) c,
\end{aligned}
$$


to $\overline{\mathcal{L}}_{\mathrm{MCG}}+\mathcal{L}_{G F 1}+\mathcal{L}_{G F 2}$ gives the effective Lagrangian density

$$
\begin{aligned}
\mathcal{L}_{\text {eff }}= & \overline{\mathcal{L}}_{E H}+2 \sigma \bar{R}+6 \partial^{\mu} \sigma \partial_{\mu} \sigma \\
& -\frac{1}{m^{2}}\left(\bar{R}^{\mu \nu} \bar{R}_{\mu \nu}-\frac{1}{3} \bar{R}^{2}\right) \\
& -\frac{1}{2 \xi_{1}}\left(\partial^{\mu} h_{\mu \nu}-\frac{1}{2} \partial_{\nu} h\right)^{2} \\
& +\frac{1}{6 \xi_{2}}\left(\frac{1}{m} \bar{R}-6 \xi_{2} m \sigma\right)^{2} \\
& +\tilde{c}^{\mu} \square c_{\mu}+2 \tilde{c}\left(\square-\xi_{2} m^{2}\right) c,
\end{aligned}
$$

where $\left(\tilde{c}^{\mu}\right) c^{\mu}$ is a vector (anti-ghost) ghost field, and $(\tilde{c}) c$ is a scalar (anti-ghost) ghost field.

We can linearize the gauge-fixing terms of the Lagrangian density (20) by writing it in the form

$$
\begin{aligned}
\mathcal{L}_{\text {eff }}= & \overline{\mathcal{L}}_{E H}+2 \sigma \bar{R}+6 \partial^{\mu} \sigma \partial_{\mu} \sigma \\
& -\frac{1}{m^{2}}\left(\bar{R}^{\mu \nu} \bar{R}_{\mu \nu}-\frac{1}{3} \bar{R}^{2}\right) \\
& -B^{\mu}\left(\partial^{\nu} h_{\mu \nu}-\frac{1}{2} \partial_{\mu} h\right) \\
& +\frac{1}{3} B\left(\frac{1}{m} \bar{R}-6 \xi_{2} m \sigma\right) \\
& +\frac{1}{2} \xi_{1} B^{\mu} B_{\mu}-\frac{1}{6} \xi_{2} B^{2} \\
& +\tilde{c}^{\mu} \square c_{\mu}+2 \tilde{c}\left(\square-\xi_{2} m^{2}\right) c,
\end{aligned}
$$

where

$B_{\mu}=\frac{1}{\xi_{1}}\left(\partial^{v} h_{\mu \nu}-\frac{1}{2} \partial_{\mu} h\right)$

is a Nakanishi-Lautrup auxiliary vector field, and

$B=\frac{1}{\xi_{2}}\left(\frac{1}{m} \bar{R}-6 \xi_{2} m \sigma\right)$

is a Nakanishi-Lautrup auxiliary scalar field.

The action corresponding to the Lagrangian density (21) is invariant under the BRST transformations

$$
\begin{aligned}
& \delta_{1} h_{\mu \nu}=\partial_{\mu} c_{\nu}+\partial_{\nu} c_{\mu}, \quad \delta_{1} c_{\mu}=0, \quad \delta_{1} \tilde{c}_{\mu}=B_{\mu}, \\
& \delta_{1} B_{\mu}=0, \quad \delta_{1} \sigma=0, \quad \delta_{1} c=0, \quad \delta_{1} \tilde{c}=0, \quad \delta_{1} B=0, \\
& \delta_{2} h_{\mu \nu}=2 m c \eta_{\mu \nu}, \quad \delta_{2} c_{\mu}=0, \quad \delta_{2} \tilde{c}_{\mu}=0, \quad \delta_{2} B_{\mu}=0, \\
& \delta_{2} \sigma=-m c, \quad \delta_{2} c=0, \quad \delta_{2} \tilde{c}=B, \quad \delta_{2} B=0 .
\end{aligned}
$$

By adding the compensating terms

$$
\begin{aligned}
\mathcal{L}_{\Omega_{1}} & =\Omega_{1}\left(-\frac{1}{2} B^{\mu} \tilde{c}_{\mu}\right)=\Omega_{1} P_{1}, \\
\mathcal{L}_{\Omega_{2}} & =\Omega_{2}\left(\frac{1}{6} B \tilde{c}\right)=\Omega_{2} P_{2},
\end{aligned}
$$

to (21), we extend the BRST invariance of the theory to include the extra BRST transformations

$\delta_{1} \xi_{1}=\Omega_{1}, \quad \delta_{1} \xi_{2}=0$,

$\delta_{2} \xi_{1}=0, \quad \delta_{2} \xi_{2}=\Omega_{2}$,

where $\Omega_{1}$ and $\Omega_{2}$ are anticommuting constant scalar sources.

The extended BRST invariance implies in the WardTakahashi identities $[16,17]$

$$
\begin{aligned}
& \Omega_{1} \frac{\partial \Gamma}{\partial \xi_{1}}+\int d^{4} x\left(\frac{\delta \Gamma}{\delta K^{\mu \nu}} \frac{\delta \Gamma}{\delta \widehat{h}_{\mu \nu}}+\frac{\delta \Gamma}{\delta \widehat{\tilde{c}}_{\mu}} \widehat{B}_{\mu}\right)=0 \\
& \Omega_{2} \frac{\partial \Gamma}{\partial \xi_{2}}+\int d^{4} x\left(\frac{\delta \Gamma}{\delta L^{\mu \nu}} \frac{\delta \Gamma}{\delta \widehat{h}_{\mu \nu}}+\frac{\delta \Gamma}{\delta L} \frac{\delta \Gamma}{\delta \widehat{\sigma}}+\frac{\delta \Gamma}{\delta \widehat{\tilde{c}}} \widehat{B}\right)=0
\end{aligned}
$$

where $K^{\mu \nu}, L^{\mu \nu}$, and $L$ are sources for the composite fields $\delta_{1} h_{\mu \nu}, \delta_{2} h_{\mu \nu}$, and $\delta_{2} \sigma$, respectively, and $\Gamma$ is the effective action. Differentiating (30) and (31) with respect to $\Omega_{1}$ and $\Omega_{2}$, respectively, and setting $\Omega_{1}=\Omega_{2}=0$, we obtain

$$
\begin{aligned}
& \frac{\partial \Gamma}{\partial \xi_{1}}-\int d^{4} x\left(\frac{\delta \Gamma\left(P_{1}\right)}{\delta K^{\mu \nu}} \frac{\delta \Gamma}{\delta \widehat{h}_{\mu \nu}}+\frac{\delta \Gamma\left(P_{1}\right)}{\delta \widehat{\tilde{c}}_{\mu}} \widehat{B}_{\mu}\right)=0, \\
& \frac{\partial \Gamma}{\partial \xi_{2}}-\int d^{4} x\left(\frac{\delta \Gamma\left(P_{2}\right)}{\delta L^{\mu \nu}} \frac{\delta \Gamma}{\delta \widehat{h}_{\mu \nu}}+\frac{\delta \Gamma\left(P_{2}\right)}{\delta L} \frac{\delta \Gamma}{\delta \widehat{\sigma}}+\frac{\delta \Gamma\left(P_{2}\right)}{\delta \widehat{\tilde{c}}} \widehat{B}\right)=0 .
\end{aligned}
$$

Finally, by differentiating (32) and (33) twice with respect to $\widehat{h}_{\mu \nu}$, and setting all sources and fields equal to zero, with the exception of $\widehat{\sigma}$ which is taken to be a constant background field, we find the Nielsen identities for the inverse propagator

$$
\begin{aligned}
& \left(\frac{\partial}{\partial \xi_{1}}\right) \frac{\delta^{2} \Gamma}{\delta \widehat{h}_{\mu \nu} \delta \widehat{h}_{\alpha \beta}}=0, \\
& \left(\frac{\partial}{\partial \xi_{2}}+C\left(\widehat{\sigma}, \xi_{2}\right) \frac{\partial}{\partial \widehat{\sigma}}\right) \frac{\delta^{2} \Gamma}{\delta \widehat{h}_{\mu \nu} \delta \widehat{h}_{\alpha \beta}}=\frac{\partial V\left(\widehat{\sigma}, \xi_{2}\right)}{\partial \widehat{\sigma}} \\
& \times \int d^{4} x \frac{\delta^{3} \Gamma\left(P_{2}\right)}{\delta L \delta \widehat{h}_{\mu \nu} \delta \widehat{h}_{\alpha \beta}},
\end{aligned}
$$

where

$C\left(\widehat{\sigma}, \xi_{2}\right)=-\int d^{4} x \frac{\delta \Gamma\left(P_{2}\right)}{\delta L}$,

and $V\left(\widehat{\sigma}, \xi_{2}\right)$ is the effective potential, which is defined by

$\Gamma[\widehat{\sigma}]=\int d^{4} x V\left(\widehat{\sigma}, \xi_{2}\right)$.

To find the Nielsen identities for the position of the complex massive pole $M^{2}$, we multiply (34) and (35) by $P_{\mu \nu, \alpha \beta}^{(2)}$ and set the external momentum $p^{2}=M^{2}$, which gives

$$
\frac{\partial M^{2}}{\partial \xi_{1}}=0
$$




$$
\begin{aligned}
& \frac{\partial M^{2}}{\partial \xi_{2}}+C\left(\widehat{\sigma}, \xi_{2}\right) \frac{\partial M^{2}}{\partial \widehat{\sigma}}=\frac{(-i) \mathscr{R}}{10 m^{2}} \frac{\partial V\left(\widehat{\sigma}, \xi_{2}\right)}{\partial \widehat{\sigma}} \\
& \times\left.\left(P_{\mu \nu, \alpha \beta}^{(2)} \int d^{4} x \frac{\delta^{3} \Gamma\left(P_{2}\right)}{\delta L \delta \widehat{h}_{\mu \nu} \delta \widehat{h}_{\alpha \beta}}\right)\right|_{k^{2}=M^{2}},
\end{aligned}
$$

where we used the inverse of the propagator (16). We can see from (38) that $M^{2}$ is independent of $\xi_{1}$. On the other hand, since there is no reason why the term between parentheses on the right-hand side of (39) should vanish, $M^{2}$ will be independent of $\xi_{2}$ only if

$\frac{\partial V\left(\widehat{\sigma}, \xi_{2}\right)}{\partial \widehat{\sigma}}=0$.

However, it follows from (20) that the zeroth order effective potential is given by

$V_{0}\left(\widehat{\sigma}, \xi_{2}\right)=6 \xi_{2} m^{2} \widehat{\sigma}^{2}$.

It is not difficult to see that the only solution of $\partial V_{0} / \partial \widehat{\sigma}$ $=0$ is the trivial $\widehat{\sigma}=0$. Thus, unless some unexpected cancellations take place in higher orders, we have

$\frac{\partial V\left(\widehat{\sigma}, \xi_{2}\right)}{\partial \widehat{\sigma}} \neq 0$

which means that $M^{2}$ depends on $\xi_{2}$. Similarly, we can show that the same is valid for $M^{* 2}$. Since we can define a gaugeinvariant $S$-matrix, such gauge-dependent poles disappear from the spectrum and unitarity is satisfied.

\section{Final remarks}

Here we presented a study on the unitarity of MCG. First, we noted that due to the presence of the unstable massive spin-2 ghost state in the linearized theory, we must use a dressed propagator perturbation expansion, which results in the emergence of a pair of complex poles in the first sheet of the energy plane. Then, by using the Nielsen identities, it was shown that the positions of the complex poles are found to be dependent on the conformal gauge-fixing parameter. This is enough to ensure that the excitations represented by the complex poles do not contribute to the gauge-invariant absorptive part of the $S$-matrix, leading to the unitarity of the theory. Therefore, we conclude that MCG is a consistent, renormalizable and unitary theory of quantum gravity.

Open Access This article is distributed under the terms of the Creative Commons Attribution 4.0 International License (http://creativecomm ons.org/licenses/by/4.0/), which permits unrestricted use, distribution, and reproduction in any medium, provided you give appropriate credit to the original author(s) and the source, provide a link to the Creative Commons license, and indicate if changes were made. Funded by $\mathrm{SCOAP}^{3}$.

\section{References}

1. F.F. Faria, Eur. Phys. J. C 76, 188 (2016)

2. F.F. Faria, Eur. Phys. J. C 77, 11 (2017)

3. D.A. Johnston, Nucl. Phys. B 297, 721 (1988)

4. F.F. Faria, Adv. High Energy Phys. 2014, 520259 (2014)

5. K. J. Barnes, Ph.D. thesis unpublished (1963)

6. R.J. Rivers, Il Nuovo Cimento 34, 387 (1964)

7. M.J.G. Veltman, Physica 29, 186 (1963)

8. I. Antoniadis, E.T. Tomboulis, Phys. Rev. D 33, 2756 (1986)

9. E.T. Tomboulis, Phys. Lett. 708, 361 (1977)

10. N.K. Nielsen, Nucl. Phys. B 101, 173 (1975)

11. C. Becchi, A. Rouet, R. Stora, Phys. Lett. B 52, 344 (1974)

12. C. Becchi, A. Rouet, R. Stora, Commun. Math. Phys. 42, 127 (1975)

13. C. Becchi, A. Rouet, R. Stora, Ann. Phys.(N.Y.) 98, 287 (1976)

14. I. V. Tyutin, Lebedev Institute Preprint, Report No: FIAN-39 (1975) (unpublished)

15. L.D. Faddeev, V.N. Popov, Phys. Lett. B 52, 29 (1967)

16. J. C. Ward, Phys. Rev. 78, 182 (1950)

17. Y. Takahashi, Il Nuovo Cimento. 6, 371 (1957) 